\title{
EASY -TO-USE MODELLING - 3D RAY FIELD PROPAGATION IN OPEN RAY MODELS
}

\section{Introduction}

Ray modelling has proven useful in a wide range of seismic applications, including survey planning, velocity inversion, map migration, synthetic seismogram generation, model-based stacking, and pre- and post-stack depth migration. Still, the spreading of modelling-based techniques relies very much on how easy modelling is to use. If modelling requires lots of special interpretation and preparation and very specialized skill to make useful results, the application is limited to the rare, special cases. On the other hand, if modelling in large can do with data made in standard processing and interpretation, and easy-to-use, efficient, and robust ray calculation is available, ray-based modelling applications may flourish. Simple ray-based applications, like map migration, may even become part of the standard, routine tools.

This paper describes a basic ray modeller that is simple, easy-to-use and robust, and can mostly do with existing data. Two basic elements are focused: robust wave field propagation and the geometrical model through which the field propagates. In particular, the interaction between propagator and model is analysed to tailor the geometrical model to this specific application. As it turns out, a tailored model is much simpler and easier to make than a general-purpose geological model.

\section{The ray field propagation}

Traditionally, ray calculation techniques have focused on the calculation of single rays between sources and receivers. The two common techniques, ray shooting and ray bending, both basically search for a single ray between one shot and one receiver, and the search is repeated to simulate surveys. Another family of ray calculation techniques are the ray field propagation techniques, where the goal is to calculate the entire wave field covered by rays from a source. Ray field calculation was first introduced in the wavefront construction technique[1],[2]. In this paper, another method, ray field segment propagation[3], is applied. The main points of this method are summarized in the following.

A ray field segment is a part of the ray field that is continuous and smooth when regarded as function of ray start direction and travel time. As the ray field propagates, new segments are made whenever interfaces are crossed, as the first derivatives of the field are discontinuous across velocity contrasts. The ray field is split into new segments only at discontinuities in value or first derivatives, thus the ray field is separated into the smallest possible number of continuous and smooth segments. Each ray field segment is represented by an ordered set of ray segments. A ray segment is a piece of a ray with no interface intersections within its interior. Each ray segment is calculated with an ordinary initial-value ray-tracer. Where the separation between adjacent ray segments is above a user-defined limit, a new segment start point is computed by interpolation, and the new ray segment is propagated and included in the ray field segment. This procedure ensures that the entire ray field is scanned within a user-defined resolution. The arrivals in receivers are calculated by interpolation within each ray field segment.

The final ray field from a shot is represented by a ray field tree with the ray field segments as nodes. The initial ray field out from the shot is the root node, and the children below each node are the ray fields that their parent is split into at interfaces. The ray field tree is a convenient, high-level representation of a complete ray field. 
As in all ray techniques, the propagation is governed by a user-defined ray code, which prescribes the wave mode $(P$ or $S)$ in the layers, and whether the rays shall be reflected or transmitted at the interfaces. The ray code may also prescribe ray field branching, a splitting into $\mathrm{P}$ and $\mathrm{S}$ and/or reflected and transmitted at some interfaces. Where branching is specified, a separate set of ray field segments is generated for each branch. An example of a simple ray field is shown in figure 1 .

The general ray field segment strategy is independent of dimension, and is equally applicable in 2D and 3D. However, the initial-value ray-tracer as well as the structure of each ray field segment depends on dimension. In 2D the ray segments are ordered in a linear sequence, while in 3D the ray segments are ordered in a triangular network with the ray segments in the corners.

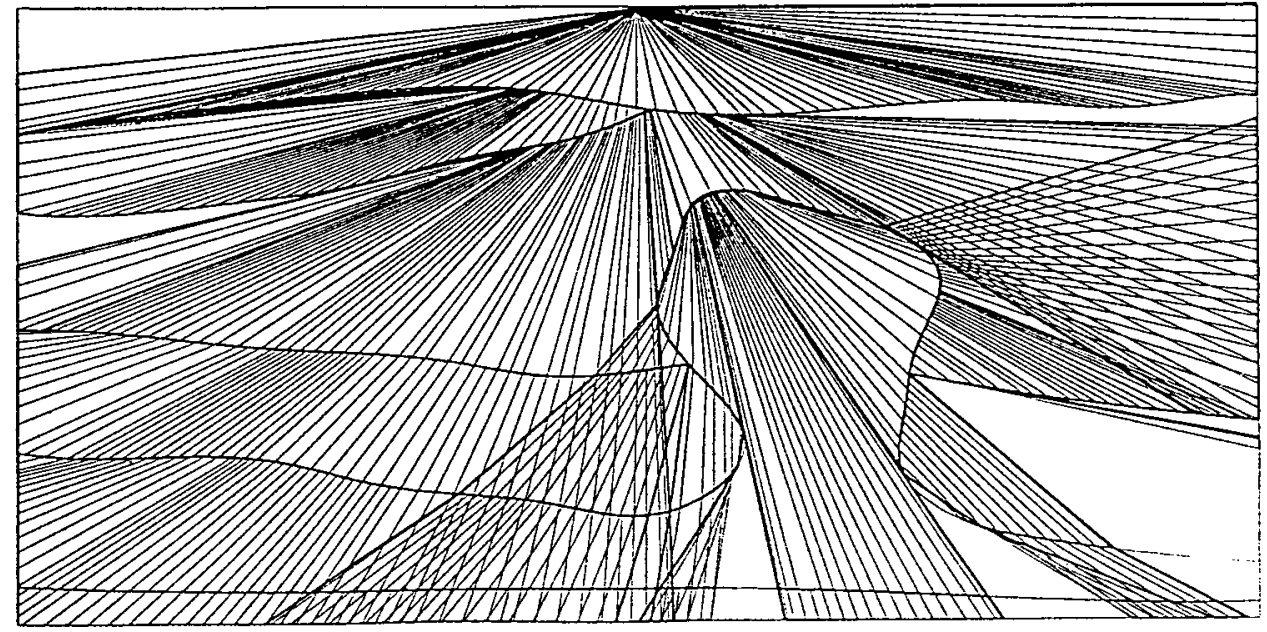

Fig.1 A vertical model cross section showing the transmitted ray field from a shot. The thin lines are rays, the bold ones are the borders of the ray field segments.

\section{Tailored model design}

The model representation is based on a detailed analysis of what the ray field propagator requires from the model. The basic calculation is propagation of a single ray segment. The ray segment starts from a known point in a known direction, and, except in the shot point, the start point is always on an interface. The ray segment propagates in a continuous velocity field until it intersects with an interface. At the interface, Snell's law is applied, and a new ray segment is started.

The ray tracing focuses on the interfaces, and this is reflected in the model representation. The basic unit is the interface. An interface is a piece of any kind of boundary (horizon, fault plane, salt dome flank). The piece must have exactly one velocity field on each side. Intersecting horizons must be split into separate interfaces (roughly) along the intersection curves. Each continuous and smooth velocity field is a separate element and is independent of the interfaces. The interfaces only refer to (point at) the velocity fields, one for each side of the interface. All interfaces that enclose the same 'block', must refer to the same velocity field for the appropriate side. Still, there is no explicit volume or block entity in the model representation, the ray propagation can do without. (If a shot is on no interface, a preliminary ray is traced to until an interface is hit, and the right velocity field is found at the interface.)

In conclusion, a sufficient model simply consists of a set of interfaces, a set of velocity fields, and a velocity field reference for each side of each interface. This general geometrical model structure is independent of dimension, while the representation of each interface and velocity field is different in 2D and 3D.

\section{The open ray model}

The geometrical model developed from the above analysis of the ray field propagation, is called the open ray model. The ray term refers to that it is tailored to ray modelling. The special-purpose design simplifies the model representation. In particular it reduces, and in many cases even eliminates, the work required to prepare a geometrical model from interpretations, mainly due to the open model concept.

The model is open in two different ways: First, it is open as to the representation of horizons and velocity fields. As 
each interface and each velocity field is independent, different representations may be applied in the same model. The set of representations may also be expanded, as the ray field propagator applies the model elements in a simple, limited and well-defined way. The application may choose the most appropriate representation individually for each model element. Usually, for simplicity and efficiency, most horizons and velocity fields are on regular grids, while steep faults and overhanging salt domes are represented by triangular networks, which are very general, but require more computations.

The model is also open in a more literal sense. There may be holes, missing parts, within the interfaces. For instance, a horizon don't have to terminate exactly at a fault plane. Inaccuracies in interpretation or limited resolution in representation may leave small holes close to the fault, still the modelling works. Conversely, a horizon may penetrate the fault plane so that small pieces of the horizons appear on the 'wrong side' of the fault. This is also accepted in the modelling. This robustness to minor inaccuracies and errors in the interfaces is the key to minimize model preparation.

The robustness is part of the ray tailoring and is due to the ray segment consistency check. It ensures that all rays obey the reciprocity principle. The consistency check is a simple comparison of the velocity fields in the start and end points of each ray segment. Recall that velocity field references are associated with each side of each interface. The velocity field on the incident side of the interface in the ray segment end point is compared with the velocity field that was found in the segment start point and used in the ray propagation. If it is the same field, the ray segment is consistent. If the fields are different, a ray traced backwards from the segment end point would have propagated through another ray field than the forward ray, and the reciprocity principle would have been violated. By default, the ray segment is rejected and removed from its ray field segment.

Inconsistent ray segments occur where there is a hole in an interface. Some rays propagate through the hole and hit next interface which is associated with another velocity field. The effect of a hole is thus that a small part of the ray field is missing locally, but there will be no invalid ray field segments. Similarly, if an interface penetrates through other ones so that there are some non-physical pieces, ray segments involving the pieces will also be rejected (see figure 2). For some interfaces it may be useful and sensible to specify action if inconsistency is detected: The interface may be regarded as invisible, and the ray is continued in the original velocity field.

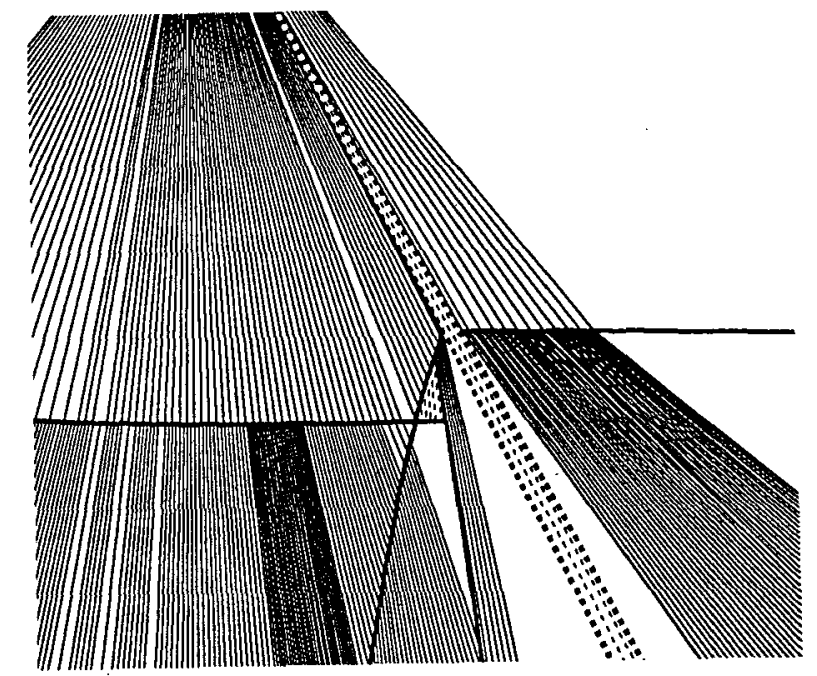

Fig. 2 The ray segment consistency check rejects ray segments that are affected by model errors. The bold, solid lines are interfaces, the thin, solid lines are accepted ray segments, while the dashed lines are rejected ones. 


\section{Applications}

Ray field propagation in open ray models is well suited to applications that require comprehensive ray modelling in complex models. Applications include:

- 3D map migration of interpreted time horizons. The open ray model concept reduces special preparation of the horizons.

- Synthetic seismogram calculations. The propagation method ensures that all arrivals in the receivers are found. The ray field branching makes the method very efficient for computation of for instance all primary reflections, as many ray field segments are shared by the different reflections, and these segments are computed only once.

- Layer-stripping velocity inversion techniques. As each ray field segment is confined to a single velocity field, segments computed in the shallower layers may be saved for reuse as the velocity fields and depth horizons are completed for the deeper layers.

- Green's function calculations for (local) 3D depth migration. Green's functions are computed are very effectively with the ray field propagator because the computation time increases only slowly with the number of target points. Re-computation after local model changes are fast because many of the ray field segments may be reused.

Selected application examples will be shown.

\section{Summary}

The main highlights of ray field propagation in open ray models are:

- Uses a simple, but general and flexible geometrical model.

- Minimizes model preparation, as minor errors and inaccuracies in the model are handled.

- Scans the entire ray field within a user-defined resolution to find all arrivals in the receivers.

- Provides a high-level representation of the ray field, with splitting into a minimum number of continuous and smooth ray field segments.

\section{References}

[1] Vinje, V., Iversen, E., and Gjøystdal, H., 1993. Traveltime and amplitude estimation using wavefront construction, Geophysics, 58, 1157-1166.

[2] Vinje, V., Iversen, E., Gjøystdal, H., and Åstebøl, K., Estimation of multivalued arrivals in 3D models using wavefront construction, To be submitted to Geophysical Prospecting, 1994

[3]Åstebøl, K., Ray Field Segment Computation - an Efficient and Flexible Wave Propagation Technique, to be presented at the 56th Meeting of the EAEG, 6 - 10 June, 1994, Vienna, Austria. 